\title{
Computer simulations of relativistic whistler-mode wave-particle interactions
}

\author{
$\operatorname{AUTHOR}(\mathrm{S}):$ \\ Omura, Y; Summers, D
}

\section{CITATION:}

Omura, Y ... [et al]. Computer simulations of relativistic whistler-mode wave-particle interactions. PHYSICS OF PLASMAS 2004, 11(7): 3530-3534

\section{ISSUE DATE:}

2004-07

URL:

http://hdl.handle.net/2433/50441

\section{RIGHT:}

Copyright 2004 American Institute of Physics. This article may be downloaded for personal use only. Any other use requires prior permission of the author and the American Institute of Physics. 


\title{
Computer simulations of relativistic whistler-mode wave-particle interactions
}

\author{
Yoshiharu Omura \\ Radio Science Center for Space and Atmosphere, Kyoto University, Uji, Kyoto 611-0011, Japan \\ Danny Summers \\ Department of Mathematics and Statistics, Memorial University of Newfoundland, St. John's, \\ Newfoundland, A1C 5S7, Canada \\ and School of Pure and Applied Physics, University of KwaZulu-Natal, Durban 4041, South Africa
}

(Received 30 October 2003; accepted 9 March 2004; published online 7 June 2004)

\begin{abstract}
Whistler-mode wave-electron interactions constitute an important physical mechanism in the Earth's magnetosphere and the radiation belts of the magnetized planets. From linear theory, an analytical result for the growth rate of electromagnetic R-mode (whistler-mode) waves in a relativistic bi-Maxwellian plasma with given temperature anisotropy is obtained. In order to test the linear theory, a one-dimensional self-consistent electromagnetic particle simulation is performed with a newly developed fully relativistic code. A major background component of isotropic cold electrons and a minor component of anisotropic hot electrons in a uniform magnetic field are assumed. Driven by the temperature anisotropy of the hot relativistic electrons, the whistler-mode waves grow initially linearly, and then nonlinearly to a level at which saturation takes place. Saturation occurs due to a combination of nonlinear trapping of resonant electrons and quasilinear relaxation of the temperature anisotropy. The initial wave growth rate obtained from the particle simulation agrees well with the growth rate predicted from linear theory. In order to reduce electrostatic fluctuations and achieve accuracy in the simulation, a large number of superparticles must be used. () 2004 American Institute of Physics. [DOI: 10.1063/1.1757457]
\end{abstract}

\section{INTRODUCTION}

There is extensive literature on whistler-mode waves in space and laboratory plasmas, e.g., see the review by Stenzel. ${ }^{1}$ Spacecraft observations of whistler-mode chorus and hiss in the Earth's magnetosphere ${ }^{2-5}$ have been made since the 1970s. Whistler-mode waves play an important role in magnetospheric physics. Early work established that whistler-mode waves can pitch-angle scatter electrons into the atmospheric loss cone, ${ }^{6}$ and act to limit the flux of radiation belt electrons. ${ }^{7}$ More recently, electron stochastic acceleration by gyroresonant wave-particle interaction with whistler-mode chorus has been suggested as a key mechanism for generating relativistic $(>1 \mathrm{MeV})$ "killer" electrons in the inner magnetosphere $(3<L<5)$ following geomagnetic storms. ${ }^{8-11}$ Whistler-mode waves have also been observed in the magnetospheres of Jupiter ${ }^{12-15}$ and Uranus, ${ }^{16}$ and in the solar wind ${ }^{17}$ upstream of Mercury, Venus, Earth, and Saturn.

First theoretical treatments of the electron R-mode (whistler-mode) instability were by Kennel and Petschek, ${ }^{7}$ Sudan, ${ }^{18}$ and Kennel and Scarf ${ }^{19}$ in the nonrelativistic regime. Trubnikov ${ }^{20}$ and Shkarofsky ${ }^{21}$ derived the general dielectric tensor in a relativistic plasma. The cyclotron maser instability in the Earth's auroral zone was analyzed by $\mathrm{Wu}$ and $\mathrm{Lee}^{22}$ and Pritchett ${ }^{23}$ in a weakly relativistic treatment. Sentman and Goertz ${ }^{24}$ applied the theory of Lerche ${ }^{25}$ to relativistic whistler-mode instability in Jupiter's inner magnetosphere. Gladd ${ }^{26}$ included relativistic effects in calculations of the temporal and spatial growth rates of whistler-mode waves in laboratory plasmas. Yoon and Davidson ${ }^{27}$ studied the whistler-mode and cyclotron maser instabilities in a relativistic plasma, and obtained an exact analytical reduction of the dispersion equation for a special choice of anisotropic distribution function. The role of relativistic effects in the generation of electron cyclotron (R-mode) radiation by anisotropic energetic electrons was examined by Wong and Goldstein. ${ }^{28}$ Schlickeiser et $a l .{ }^{29}$ analyzed the whistler-mode dispersion relation in a relativistic Maxwellian plasma.

Building on earlier work by Lerche, ${ }^{25}$ Montgomery and Tidman, ${ }^{30}$ and Melrose, ${ }^{31}$ Xiao et al. ${ }^{32}$ obtained an explicit formula for the growth rate of electromagnetic R-mode waves in a relativistic plasma. Xiao et al. ${ }^{32}$ found that the growth rates calculated from fully relativistic theory are substantially smaller than those calculated using the nonrelativistic approximation. Moreover, differences resulting from relativistic and nonrelativistic treatments become noticeable at relatively small electron thermal energies (a few keV), and became significant at thermal energies exceeding $100 \mathrm{keV}$. In the present paper, we test the linear growth rate formulation of Xiao et al. ${ }^{32}$ by means of a self-consistent wave-particle simulation. The simulation is carried out by means of a newly developed, fully relativistic, one-dimensional particle code. In Sec. II we apply the linear theory of Xiao et al. ${ }^{32}$ to obtain the growth rate for electromagnetic R-mode waves in a relativistic anisotropic bi-Maxwellian plasma. We describe the particle simulation and our numerical results in Sec. III, and in Sec. IV we state our conclusions. 


\section{LINEAR THEORY}

Consider an infinite homogeneous plasma immersed in a uniform magnetic field assumed to be in the $z$-direction, $\mathbf{B}_{o}$ $=B_{o} \hat{\mathbf{z}}$. Suppose the plasma comprises a dominant cold electron population and a minor hot electron population, together with background neutralizing ions. Let the total electron distribution $f\left(p_{\|}, p_{\perp}\right)$ take the form,

$$
f=f_{0}+\nu_{h} f_{1},
$$

where $f_{0}=\delta\left(p_{\|}\right) \delta\left(p_{\perp}\right) /\left(2 \pi p_{\perp}\right)$ is the cold electron distribution function, with $\delta$ the Dirac delta-function; $f_{1}$ is the hot electron distribution function to be prescribed, and $\nu_{h}$ $=N_{1} / N_{0}(\ll 1)$, where $N_{1}$ and $N_{0}$ are the respective number densities of the hot and cold electron populations; $p=\gamma v$ is the relativistic momentum per unit mass with components $p_{\|}=\gamma v_{\|}, p_{\perp}=\gamma v_{\perp}$, respectively, parallel and perpendicular to the ambient magnetic field; $\gamma=\left(1-v^{2} / c^{2}\right)^{-1 / 2}=(1$ $\left.+p^{2} / c^{2}\right)^{1 / 2}$ (with $v^{2}=v_{\|}^{2}+v_{\perp}^{2}$ and $p^{2}=p_{\|}^{2}+p_{\perp}^{2}$ ); $v$ is the particle speed, and $c$ is the speed of light. We assume an electromagnetic wave field $\sim e^{i(k z-\omega t)}$, with real wave number $k$, and complex wave frequency $\omega=\omega_{r}+i \omega_{i}$. Then the relativistic dispersion equation ${ }^{25,30,32}$ for small-amplitude, linear R-mode electromagnetic waves propagating parallel to the background magnetic field is

$$
\begin{aligned}
k^{2}= & \frac{\omega^{2}}{c^{2}}-\frac{\omega \omega_{p e}^{2}}{c^{2}\left(\omega-\Omega_{e}\right)} \\
& +\pi \nu_{h} \frac{\omega_{p e}^{2} \omega}{c^{2}} \int_{-\infty}^{\infty} d p_{\|} \int_{o}^{\infty} d p_{\perp} \frac{p_{\perp}^{2} \hat{H} f_{1}}{\gamma \omega-\Omega_{e}-k p_{\|}},
\end{aligned}
$$

where $\Omega_{e}=e B_{o} / m_{e}$ is the electron gyrofrequency, $\omega_{p e}$ $=\left(N_{o} e^{2} /\left(\epsilon_{o} m_{e}\right)\right)^{1 / 2}$ is the electron plasma frequency, $e$ is the electronic charge, and $m_{e}$ is the electron rest mass. The operator $\hat{H}$ in (2) is given by

$$
\hat{H}=\frac{\partial}{\partial p_{\perp}}+\frac{k}{\gamma \omega}\left(p_{\perp} \frac{\partial}{\partial p_{\|}}-p_{\|} \frac{\partial}{\partial p_{\perp}}\right) .
$$

The poles of the integrand in (2), given by

$$
\gamma \omega-\Omega_{e}-k p_{\|}=0,
$$

identify the momenta of the resonant particles.

We assume the bi-Maxwellian distribution, ${ }^{33,34}$

$$
f^{M}\left(p_{\|}, p_{\perp}\right)=\frac{1}{\pi^{3 / 2} a_{\perp}^{2} a_{\|}} e^{-\left(p_{\|}^{2} / a_{\|}^{2}+p_{\perp}^{2} / a_{\perp}^{2}\right)},
$$

where $a_{\|}$and $a_{\perp}$ are parallel and perpendicular thermal momenta, which can be regarded as the respective parallel and perpendicular "thermal speeds." The thermal anisotropy is defined as

$$
A=\frac{a_{\perp}^{2}}{a_{\|}^{2}}-1
$$

and provides a source of free energy for wave growth; Xiao et al. $^{32}$ obtained a general result for the wave growth/ damping rate $\omega_{i}$ from the dispersion equation (2) for any hot distribution function $f_{1}$. In the case that $f_{1}$ is bi-Maxwellian, $f_{1}=f^{M}$, the result can be expressed in the form,

$$
\begin{aligned}
\frac{\omega_{i}}{\Omega_{e}}= & \frac{\pi \widetilde{\eta}_{\mathrm{rel}}}{\alpha\left[2\left(\frac{\omega}{\Omega_{e}}\right)+\frac{1}{\alpha} \frac{1}{\left(\frac{\omega}{\Omega_{e}}-1\right)^{2}}\right]} \\
& \times\left\{\widetilde{A}_{\mathrm{rel}}-\frac{\frac{\omega}{\Omega_{e}}}{\left(1-\frac{\omega}{\Omega_{e}}\right)}\right\},
\end{aligned}
$$

where

$$
\begin{aligned}
\widetilde{A}_{\mathrm{rel}}=\frac{A}{\left[\left(\frac{\omega}{k c}\right)-\frac{\Omega_{e}}{k c}\right]} \frac{\int_{0}^{\infty} \frac{d x \delta x^{3} e^{-Q}}{\left.\int_{0}^{\infty} \frac{d x \cdot x^{3} e^{-Q}}{c k} \frac{\delta}{y}\right]}}{\left.1-\left(\frac{\omega}{c k}\right) \frac{\delta}{y}\right]} \\
\widetilde{\eta}_{\mathrm{rel}}=\frac{2 \nu_{h}}{\sqrt{\pi} \frac{1}{b_{\perp}^{4} b_{\|}}\left(\frac{\Omega_{e}}{k c}-\frac{\omega}{k c}\right) \int_{o}^{\infty} d x x^{3} e^{-Q},} \\
y=\frac{-1+\left(\frac{c k}{\omega}\right)\left[\left\{\left(\frac{c k}{\omega}\right)^{2}-1\right\}\left(1+x^{2}\right)\left(\frac{\omega}{\Omega_{e}}\right)^{2}+1\right]^{1 / 2}}{\left\{\left(\frac{c k}{\omega}\right)^{2}-1\right\}\left(\frac{\omega}{\Omega_{e}}\right)}, \\
\delta=y\left(\frac{\omega}{k c}\right)-\frac{\Omega_{e}}{k c}, \quad Q=\frac{\delta^{2}}{b_{\|}^{2}}+\frac{x^{2}}{b_{\perp}^{2}}, \\
\alpha=\frac{\Omega_{e}^{2}}{\omega_{p e}^{2}}, \quad b_{\|}=\frac{a_{\|}}{c}, \quad b_{\perp}=\frac{a_{\perp}}{c} .
\end{aligned}
$$

In expressions (7)-(8), $\omega$ means the real wave frequency $\omega_{r}$; $\widetilde{A}_{\text {rel }}$ is the relativistic pitch-angle anisotropy of the resonant particles, and $\widetilde{\eta}_{\text {rel }}$ is the fraction of the relativistic particle distribution near resonance. Result (7) gives the wave growth/damping rate $\omega_{i}$ as a function of wave frequency since the wave number $k$ can be eliminated by use of the (real) dispersion relation for R-mode (whistler-mode) waves,

$$
\left(\frac{c k}{\omega}\right)^{2}=1+\frac{1}{\alpha \frac{\omega}{\Omega_{e}}\left(1-\frac{\omega}{\Omega_{e}}\right)},
$$

where the cold-plasma parameter $\alpha$ is defined in (8).

\section{COMPUTER SIMULATIONS}

In order to incorporate the relativistic dynamics of the particles, we have modified the one-dimensional electromagnetic particle simulation code KEMPO1 of Omura and Matsumoto, ${ }^{35}$ where Maxwell's equations and the equations of motion for a large number of particles are solved in a one-dimensional system. The current density is computed from the motion of the particles, modifying the electromagnetic fields. The charge density is computed only once from the initial particle 
(a)

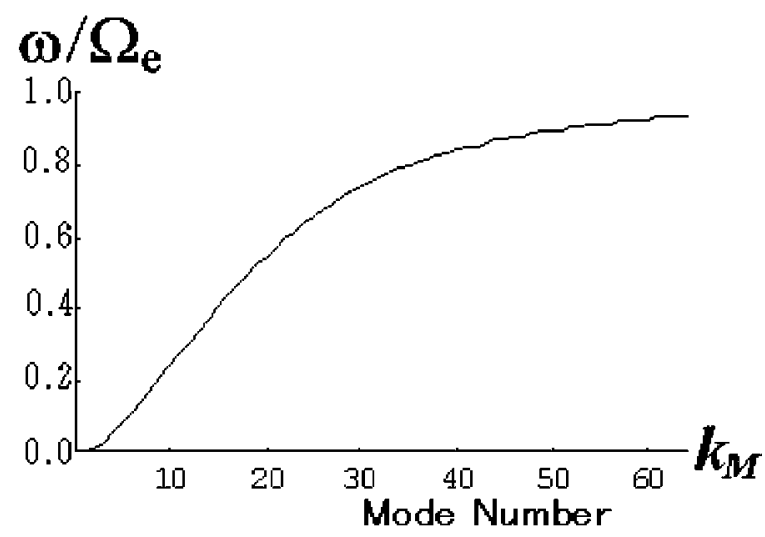

(b)

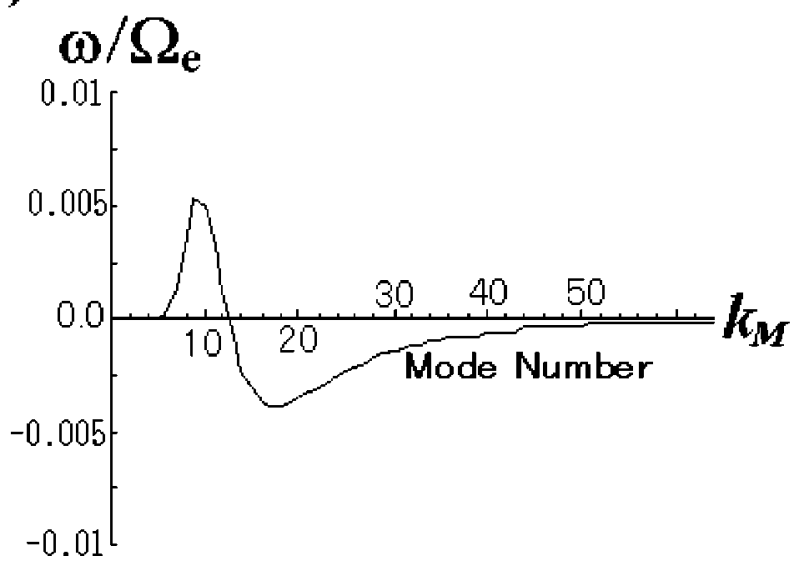

FIG. 1. (a) Whistler-mode dispersion curve (9) for $\alpha=1$. (b) Whistler-mode wave growth/damping rate (7) for $\alpha=1, b_{\|}=0.6, b_{\perp}=2.08, A=11.02$. The mode number $k_{M}$ is given by $k_{M}=k / k_{\min }$, where $k_{\min }=0.0613 \Omega_{e} / c$ is the minimum wave number in the simulation code.

positions, and the initial electrostatic field is obtained from Poisson's equation. In the subsequent time steps, the electric field, modified by the current densiy, automatically satisfies Poisson's equation, because the current density is calculated so as to satisfy the continuity equation of the charge density. We assume a one-dimensional system with periodic boundaries, where particles exiting the right boundary re-enter the system from the left boundary, and vice-versa. A detailed technical guide to KEMPO1 is provided in Chap. 2 of Ref. 35 .

The essential part of the modification to the code relates to the subroutine VELCTY in which the velocity of the particles is advanced in time under the influence of the electromagnetic fields. We solve the relativistic equation of motion,

$$
\frac{d}{d t}\left(\gamma m_{e} \mathbf{v}\right)=-e(\mathbf{E}+\mathbf{v} \times \mathbf{B}),
$$

for an electron in an electric field $\mathbf{E}$ and magnetic field $\mathbf{B}$, where $\gamma=\left(1-v^{2} / c^{2}\right)^{-1 / 2}$ is the Lorentz factor.

By setting $\mathbf{p}=\gamma \mathbf{v}$ and $\mathbf{B}_{\text {rel }}=\mathbf{B} / \gamma$, we transform Eq. (10) into the "nonrelativistic" form,

$$
\frac{d \mathbf{p}}{d t}=-\frac{e}{m_{e}}\left(\mathbf{E}+\mathbf{p} \times \mathbf{B}_{\mathrm{rel}}\right) .
$$

\section{$\left(\mathbf{B}_{\mathrm{w}} / \mathbf{B}_{\mathrm{O}}\right)^{2}$}

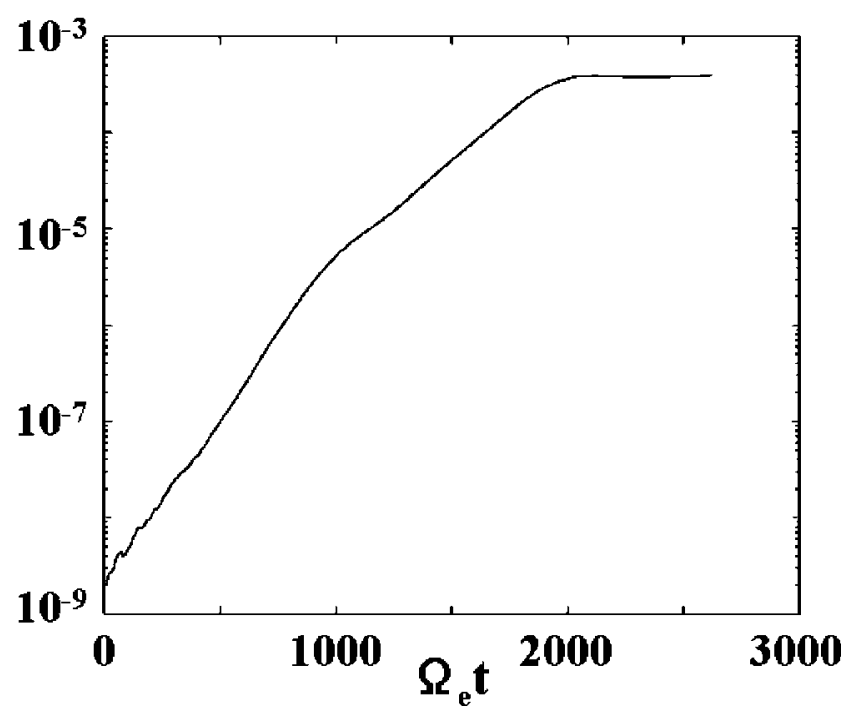

FIG. 2. Time history of normalized wave magnetic field energy.

We then solve Eq. (11) for $\mathbf{p}$ using the Buneman-Boris method, as prescribed in the original code. To transform back to the velocity $\mathbf{v}$, we use

$$
\mathbf{v}=\mathbf{p} /\left(1+p^{2} / c^{2}\right)^{1 / 2} \text {. }
$$

The hot electron component is assumed to take the biMaxwellian form (5). We perform a simulation run with strongly relativistic parameters with thermal speeds $b_{\|}$ $=a_{\|} / c=0.6, b_{\perp}=a_{\perp} / c=2.08$, corresponding to the anisotropy $A=11.02$. The average thermal energy of the electrons is $708 \mathrm{keV}$. We set the cold-plasma parameter $\alpha=\Omega_{e}^{2} / \omega_{p e}^{2}$ $=1$, and the density ratio of the hot component to the cold component $\nu_{h}=0.01$. The strong temperature anisotropy induces instability of whistler-mode waves whose growth rate can be predicted by the linear theory outlined in Sec. II. In Fig. 1 we plot in the upper panel the dispersion curve for whistler-mode waves given by Eq. (9), and, in the lower panel, the linear growth/damping rate calculated from results (7) and (9).

The dominant cold electron population is assumed to be isotropic with thermal speeds $b_{\|}=b_{\perp}=0.025$. The number of superparticles assigned to represent each of the cold electron and hot anisotropic electron populations is set to be 8388608 . The momenta of these particles are initialized by a set of normal random numbers so that they form the bi-Maxwellian distribution (5). Transformation by Eq. (12) gives the initial velocities of the particles from the momenta. The onedimensional simulation system contains 4096 grid points with the grid spacing $\delta x=0.025 c / \Omega_{e}$. The difference forms of Maxwell's equations are advanced 131072 times using the time-step $0.02 / \Omega_{e}$. Thus, we trace the evolution of the instability over the time period $2621 / \Omega_{e}$. The minimum wave number corresponding to one wavelength in the simulation system is $k_{\min }=0.0613 \Omega_{e} / c$.

In Fig. 2 we plot the time history of the wave magnetic field energy for the total period of the simulation run. Wave growth occurs during the initial phase from $t=0$ to $t$ 


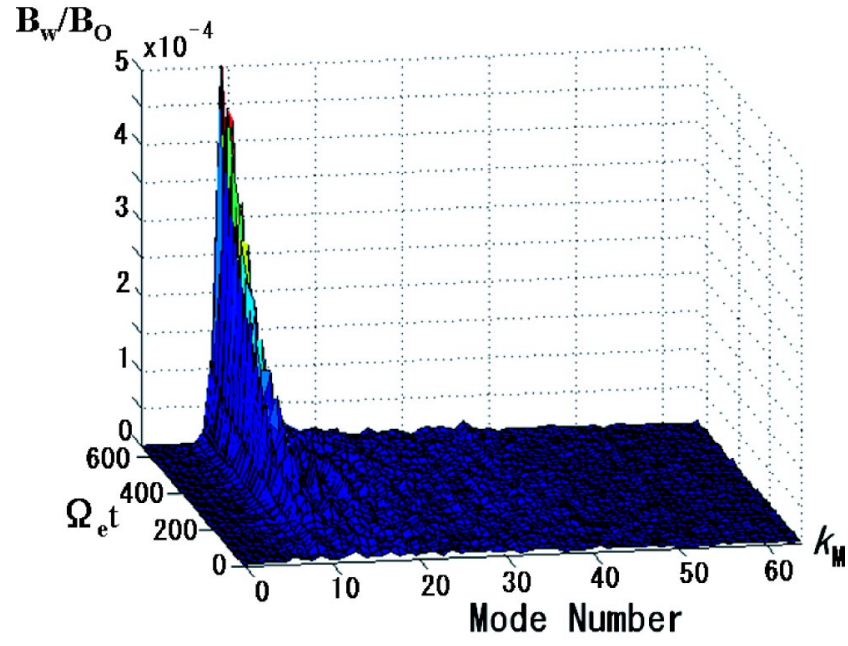

FIG. 3. Time evolution of $k$-spectra of wave magnetic field.

$=800 / \Omega_{e}$. During the initial phase, the average growth rate calculated from the slope of the wave magnetic field energy profile, is given by $\gamma_{\mathrm{sim}} / \Omega_{e}=0.004$. The growth rate predicted by the linear theory for the most unstable mode is $\omega_{i} / \Omega_{e}=0.0054$. The slight discrepancy is due to the diffusion of particles induced by enhanced thermal fluctuations that are generated during the particle simulation. In the simulation code the longitudinal electric field $E_{x}$ is calculated from Poisson's equation in the one-dimensional system. Because of the limited number of superparticles within the Debye length, namely 2048, there arise enhanced electrostatic fluctuations in the direction parallel to the magnetic field. These fluctuations diffuse the resonant electrons, thereby generating a resonant transverse current that excites whistlermode waves. The influence of the electrostatic fluctuations can be reduced by increasing the number of superparticles. A few simulations were attempted employing a larger number of superparticles, and it was found that the growth rates obtained approach the value predicted by the linear theory.

The time evolution of the wavenumber spectra of the magnetic field is shown in Fig. 3. The most unstable mode in the initial phase is mode 9 , given by $k_{9}=0.55 \Omega_{e} / c$. This wave number agrees with that corresponding to the largest growth rate illustrated in Fig. 1.

We find from Fig. 2 that during the time period from $t$ $=1000 / \Omega_{e}$ to $t=1800 / \Omega_{e}$ the growth rate decreases to $0.002 \Omega_{e}$. The instability saturates at $t=2100 / \Omega_{e}$. The decrease in growth rate and the subsequent saturation is due to the diffusion of resonant relativistic electrons. We plot the initial and final velocity distributions in the logarithmic scale in the upper and lower panels of Fig. 4. It is noted that the bi-Maxwellian distribution in momentum $\left(p_{\|}, p_{\perp}\right)$-space is strongly deformed in velocity $\left(v_{\|}, v_{\perp}\right)$-space at relativistic speeds as a result of the Jacobian transformation (a function of $\gamma$ ) between these spaces. After the initial particle diffusion associated with mode 9, quasilinear diffusion takes place, shifting from the most dominant mode subsequently to lower numbers, i.e., modes 8 and 7 . The diffusion curve is approximately an ellipse in $\left(v_{\|}, v_{\perp}\right)$-space. The phase velocities of modes 9,8 , and 7 are respectively $0.36 \mathrm{c}, 0.33 \mathrm{c}$, and $0.30 \mathrm{c}$. It
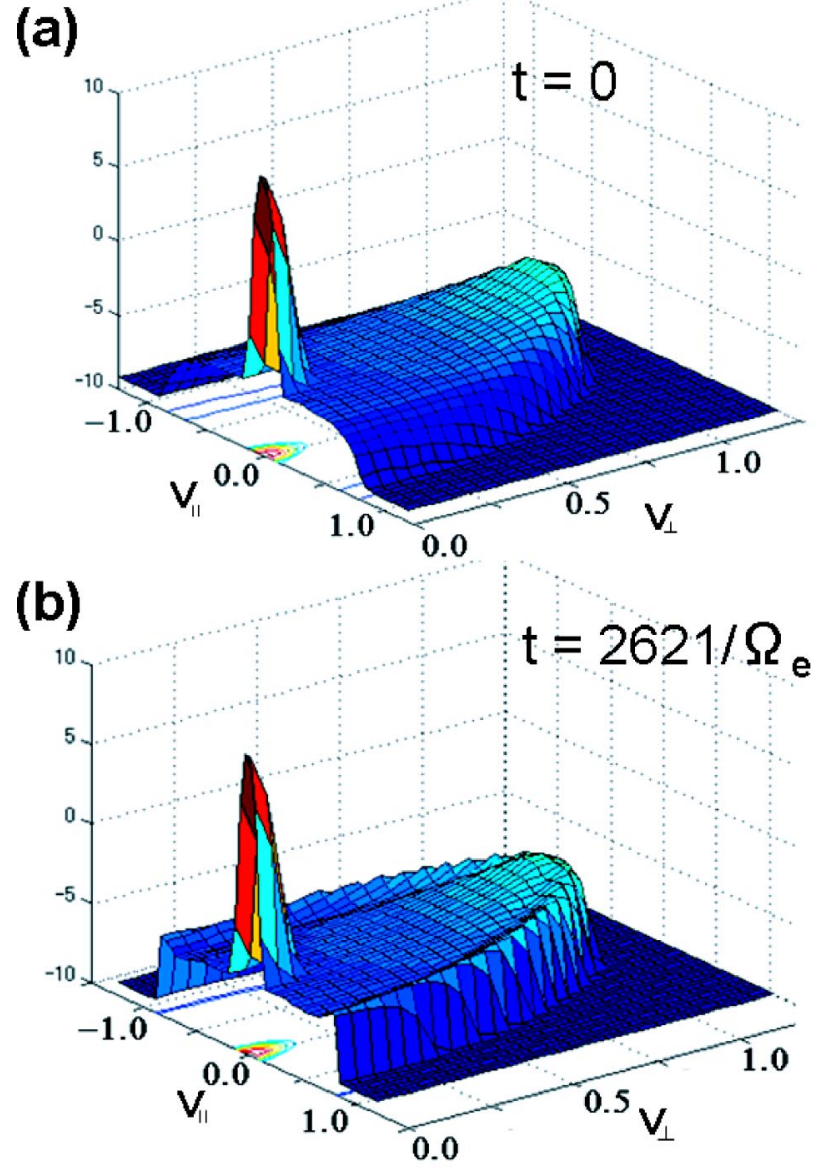

FIG. 4. (a) Initial velocity distribution (at $t=0$ ). (b) Final velocity distribution (at $t=2621 / \Omega_{e}$ ). The vertical axis represents the logarithm of the distribution function in an arbitrary scale. A slightly brighter color scale is used in the other side of the surface of the velocity distribution function.

should be noted that both forward-traveling and backwardtraveling waves are excited in the present system. The waves are driven by the temperature anisotropy of the hot electrons, in the complete absence of drift between the hot and cold electron populations. Pitch-angle diffusion in the positive velocity range is caused by the backward-traveling waves with negative phase velocities, and pitch-angle diffusion in the negative velocity range by the forward-traveling waves.

To trace the trajectories of individual particles in velocity space, we produce phase space plots of a large number of particles randomly sampled from the two sets of 8388608 particles representing the cold and hot relativistic electron populations. The results are shown in Fig. 5, in which the upper panel corresponds to the initial state, and the lower panel to the final state, $t=2621 / \Omega_{e}$. The growing whistlermode waves diffuse the particles to form a shell-like distribution, with a clearly enhanced outer edge occurring at speeds close to the speed of light.

\section{CONCLUDING REMARKS}

(1) We have derived an explicit formula for the linear growth rate of small-amplitude, R-mode (whistler-mode) electromagnetic waves in a relativistic bi-Maxwellian plasma with given thermal anisotropy. 
(a)

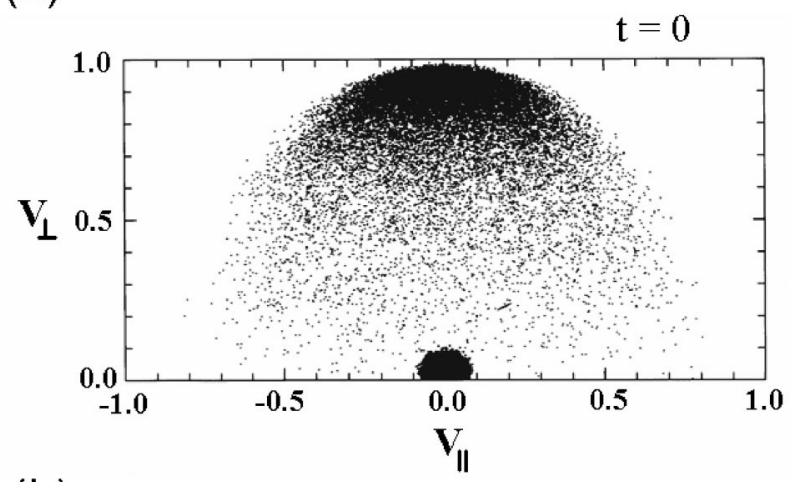

(b)

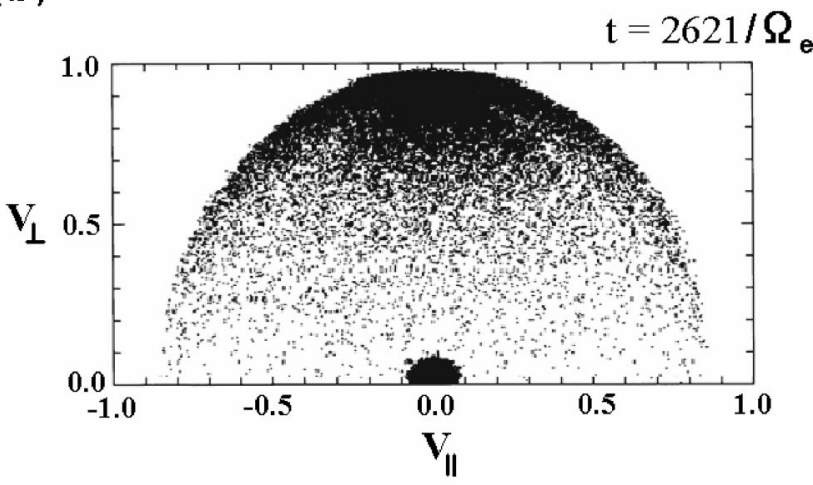

FIG. 5. (a) Initial phase space plot (at $t=0$ ). (b) Final phase space plot (at $\left.t=2621 / \Omega_{e}\right)$.

(2) Computer simulations employing a fully relativistic onedimensional electromagnetic particle code have been carried out, and a good agreement is found between the initial wave growth rate for the simulation run, and the growth rate predicted from linear theory.

(3) As a result of the limited number of superparticles used in the simulation, enhanced electrostatic fluctuations are generated that result in a reduction in the linear growth rate for the whistler-mode waves. Increasing the number of superparticles produces a decrease in the electrostatic fluctuations generated, and leads to growth rates that approach the linear theoretical result.

\section{ACKNOWLEDGMENTS}

This work was supported by the grant-in-aid 13440143 of the Ministry of Education, Science, Sports, and Culture of Japan. The project was completed when D.S. was Visiting Professor at the Radio Science Center for Space and Atmosphere (RASC), Kyoto University, Japan; D.S. thanks RASC for its warm hospitality. D.S. also acknowledges support from the Natural Sciences and Engineering Research Council of Canada under Grant No. A-0621. The computer simulations were carried out on the AKDK computer system at RASC, Kyoto University.

${ }^{1}$ R. L. Stenzel, J. Geophys. Res. 104, 14379 (1999).

${ }^{2}$ B. T. Tsurutani and E. J. Smith, J. Geophys. Res. 79, 118 (1974).

${ }^{3}$ M. Hayakawa and S. S. Sazhin, Planet. Space Sci. 40, 1325 (1992).

${ }^{4}$ N. P. Meredith, R. B. Horne, and R. R. Anderson, J. Geophys. Res. 106, 13165 (2001).

${ }^{5}$ O. Santolik, D. A. Gurnett, J. S. Pickett, M. Parrot, and N. CornilleauWehrlin, J. Geophys. Res. 108, A7, 1278, doi:10.1029/2002JA009791 (2003).

${ }^{6}$ L. R. Lyons, R. M. Thorne, and C. F. Kennel, J. Geophys. Res. 77, 3455 (1972).

${ }^{7}$ C. F. Kennel and H. E. Petschek, J. Geophys. Res. 71, 1 (1966).

${ }^{8}$ D. Summers, R. M. Thorne, and F. Xiao, J. Geophys. Res. 103, 20487 (1998).

${ }^{9}$ D. Summers and C. Ma, J. Geophys. Res. 105, 2625 (2000).

${ }^{10}$ D. Summers, C. Ma, N. P. Meredith, R. B. Horne, R. M. Thorne, D. Heynderickx, and R. R. Anderson, Geophys. Res. Lett. 29, 24, 2174 doi:10.1029/2002GL016039 (2002).

${ }^{11}$ D. Summers, C. Ma, N. P. Meredith, R. B. Horne, R. M. Thorne, and R. R. Anderson, J. Atmos. Sol.-Terr. Phys. 66, 133 (2004).

${ }^{12}$ D. A. Gurnett and F. L. Scarf, in Physics of the Jovian Magnetosphere, edited by A. J. Dessler (Cambridge University Press, Cambridge, 1983), p. 285.

${ }^{13}$ N. Lin, P. J. Kellogg, J. P. Thiessen, D. Lengyel-Frey, B. T. Tsurutani, and J. L. Phillips, J. Geophys. Res. 99, 23527 (1994).

${ }^{14}$ Y. Hobara, S. Kanemaru, M. Hayakawa, and D. A. Gurnett, J. Geophys. Res. 102, A4, 7115 (1997).

${ }^{15}$ W. S. Kurth, D. A. Gurnett, A. M. Persoon, A. Roux, S. J. Bolton, and C. J. Alexander, Planet. Space Sci. 49, 345 (2001).

${ }^{16}$ W. S. Kurth, D. A. Gurnett, and F. L. Scarf, J. Geophys. Res. 91, 11958 (1986).

${ }^{17}$ D. S. Orlowski and C. T. Russell, Adv. Space Res. 16, 137 (1995).

${ }^{18}$ R. N. Sudan, Phys. Fluids 6, 57 (1963).

${ }^{19}$ C. F. Kennel and F. L. Scarf, J. Geophys. Res. 73, 6149 (1968).

${ }^{20} \mathrm{~B}$. A. Trubnikov, in Plasma Physics and the Problem of Controlled Thermonuclear Reactions, edited by M. A. Leontovich (Pergamon, New York, 1959), p. 122.

${ }^{21}$ I. P. Shkarofsky, Phys. Fluids 9, 561 (1966).

${ }^{22}$ C. S. Wu and L. C. Lee, Astrophys. J. 230, 621 (1979).

${ }^{23}$ D. L. Pritchett, J. Geophys. Res. 89, 8957 (1984).

${ }^{24}$ D. Sentman and C. K. Goertz, J. Geophys. Res. 83, 3151 (1978).

${ }^{25}$ I. Lerche, Astrophys. J. 147, 689 (1967).

${ }^{26}$ N. T. Gladd, Phys. Fluids 26, 974 (1983).

${ }^{27}$ P. H. Yoon and R. C. Davidson, Phys. Rev. A 35, 2619 (1987).

${ }^{28}$ H. K. Wong and M. L. Goldstein, J. Geophys. Res. 99, 235 (1994).

${ }^{29}$ R. Schlickeiser, H. Fichtner, and M. Kneller, J. Geophys. Res. 102, 4725 (1997).

${ }^{30}$ D. C. Montgomery and D. A. Tidman, Plasma Kinetic Theory (McGrawHill, New York, 1964).

${ }^{31}$ D. B. Melrose, Plasma Astrophysics (Gordon and Breach, New York, 1980), Vol. 1.

${ }^{32}$ F. Xiao, R. M. Thorne, and D. Summers, Phys. Plasmas 5, 2489 (1998).

${ }^{33}$ M. Bornatici, G. Chiozzi, and P. DeChiara, J. Plasma Phys. 44, 319 (1990).

${ }^{34}$ K. T. Tsang, Phys. Fluids 27, 1659 (1984).

${ }^{35}$ Y. Omura and H. Matsumoto, in Computer Space Plasma Physics, edited by H. Matsumoto and Y. Omura (Terra Scientific, Tokyo, 1993), p. 21. 